10.3

\title{
Параметрическое усиление магнитоакустических колебаний В структуре ферромагнетик-пьезоэлектрик
}

\author{
() Д.А. Бурдин, Д.В. Чашин, Н.А. Экономов, Ю.К. Фетисов \\ МИРЭА - Российский технологический университет, Москва, Россия \\ E-mail: phantastic@mail.ru
}

Поступило в Редакцию 18 ноября 2019г.

В окончательной редакции 18 ноября 2019г.

Принято к публикации 22 ноября 2019г.

\begin{abstract}
Обнаружено параметрическое усиление магнитоакустических колебаний в дисковом резонаторе, содержащем ферромагнитный слой $\mathrm{FeBSiC}$ и пьезоэлектрический слой цирконата-титаната свинца. Колебания с частотой $3.08 \mathrm{kHz}$ возбуждались и регистрировались с помощью двух катушек с ортогональными осями. Накачка проводилась электрическим полем с удвоенной частотой, приложенным к пьезоэлектрическому слою. Усиление колебаний возникает за счет изменения жесткости структуры под действием электрического поля. Показано, что коэффициент усиления можно изменять с помощью постоянного магнитного поля, приложенного к структуре.
\end{abstract}

Ключевые слова: параметрическое усиление, магнитоакустические колебания, ферромагнетик, пьезоэлектрик.

DOI: 10.21883/PJTF.2020.04.49053.18116

Параметрическое усиление наблюдается в линейных резонансных системах при возбуждении колебаний с частотой $f$, близкой к частоте $f_{0}$ собственного резонанса системы, и одновременном воздействии на систему внешней накачки с частотой $2 f / k$ (где $k=1,2, \ldots$ ), которая изменяет какой-либо параметр системы, задающий ее собственную частоту. Параметрическое усиление реализовано в $L C$-резонаторах в диапазоне радиочастот [1], ферромагнитных резонаторах в сверхвысокочастотном диапазоне [2], оптических резонаторах в оптическом диапазоне [3]. В работах [4-6] наблюдалось параметрическое усиление колебаний микробалок, используемых в атомно-силовых микроскопах.

В настоящей работе обнаружено и исследовано параметрическое усиление магнитоакустических колебаний в дисковом резонаторе, содержащем ферромагнитный (ФМ) и пьезоэлектрический (ПЭ) слои. Резонатор представлял собой диск диаметром $R=8 \mathrm{~mm}$, содержащий ФМ-слой аморфного ферромагнетика $\mathrm{FeBSiC}$ толщиной $a_{m}=20 \mu \mathrm{m}$ (Metglas $\left.2605 \mathrm{SA} 1\right)$ и ПЭ-слой цирконата-титаната свинца $\mathrm{PbZr}_{0.52} \mathrm{Ti}_{0.48} \mathrm{O}_{3}$ (PZT) толщиной $a_{p}=100 \mu \mathrm{m}$. Магнитострикция насыщения ФМслоя достигала $\lambda_{S} \approx 23 \cdot 10^{-6}$ в касательном магнитном поле $H_{S} \sim 8 \mathrm{kA} / \mathrm{m}$. На поверхности слоя РZТ были нанесены Ag-электроды толщиной $\sim 2 \mu \mathrm{m}$, и он был поляризован перпендикулярно плоскости в постоянном электрическом поле $\sim 15 \mathrm{kV} / \mathrm{cm}$. Пьезомодуль РZT равнялся $d_{31} \approx 175 \mathrm{pm} / \mathrm{N}$. Слои структуры были соединены под прессом слоем эпоксидного клея толщиной $\sim 2 \mu \mathrm{m}$.

Резонатор был помещен внутрь плоских, вставленных одна в другую электромагнитных катушек с взаимно перпендикулярными осями, как показано на вставке к рис. 1. Параллельно плоскости структуры вдоль оси $x$ прикладывалось постоянное поле $H=0-8 \mathrm{kA} / \mathrm{m}$. На внутреннюю катушку с выхода двухканального генератора АКІР3410 подавалось напряжение $U(f)$, которое создавало в образце возбуждающее магнитное поле $h \cos (2 \pi f t)$ с амплитудой $h=0.8 \mathrm{~A} / \mathrm{m}$ и частотой $f=0.1-10 \mathrm{kHz}$. Это поле посредством магнитострикции возбуждало в резонаторе магнитоакустические колебания. Внешняя измерительная катушка использовалась для регистрации амплитуды колебаний. На электроды слоя РZT со второго выхода генератора подавалось гармоническое напряжение $U_{p}(2 f)$ с удвоенной частотой, которое создавало в слое РZТ электрическое поле накачки $e \cos (4 \pi f t)$ с амплитудой до $e=500 \mathrm{~V} / \mathrm{cm}$. Генератор позволял регулировать сдвиг фаз $\varphi$ между возбуждающим напряжением $U$ и напряжением накачки $U_{p}$ в пределах $0-2 \pi$. Амплитуда напряжения $u$, генерируемого измерительной катушкой, регистрировалась с помощью FFT Network Analyzer SR770 при различных значениях частоты $f$ и магнитных полей $h$ и $H$.

На рис. 1 приведена зависимость напряжения $u$ от частоты $f$ возбуждающего магнитного поля амплитудой $h=0.8 \mathrm{~A} / \mathrm{m}$ в отсутствие электрической накачки $(e=0)$. Пики напряжения с частотами $f_{1} \approx 3.26 \mathrm{kHz}$ и $f_{2} \approx 5.86 \mathrm{kHz}$ и добротностями $Q_{1,2} \approx 30$ по уровню 0.7 соответствуют, как показало моделирование с использованием пакета COMSOL Multiphysics, возбуждению низших мод изгибных колебаний резонатора. Благодаря ортогональной ориентации осей возбуждающей и измерительной катушек амплитуда наводки на частотах вдали от резонансов не превышала $\sim 5 \mu \mathrm{V}$. Стрелками на рисунке обозначены положения частоты $f_{s}$ возбуждающего магнитного поля и частоты $f_{p}=2 f_{s}$ электрического поля накачки при последующем исследовании параметрического усиления. 


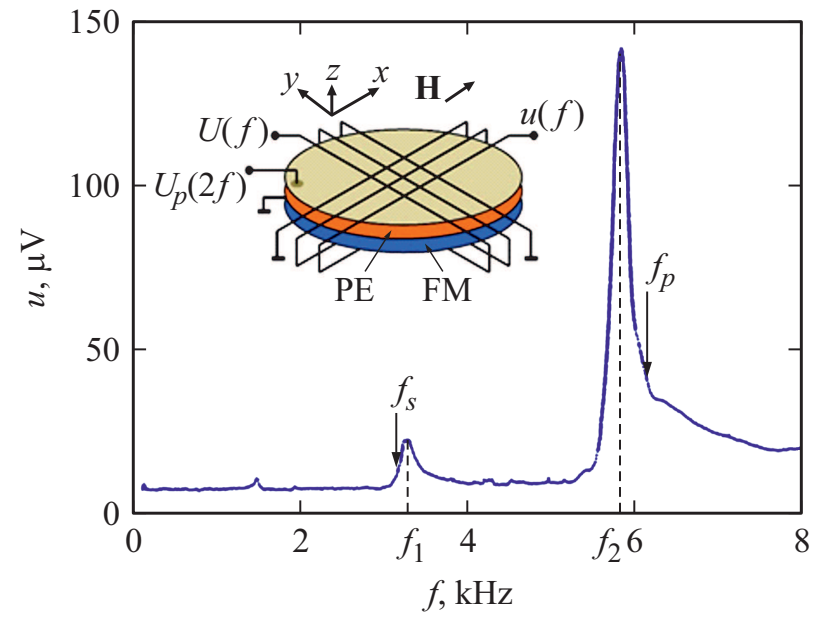

Рис. 1. Зависимость напряжения $u$ с приемной катушки от частоты $f$ возбуждающего магнитного поля. На вставке схематически изображена дисковая ФМ-ПЭ-структура.

На рис. 2 показан спектр частот напряжения $u(f)$, генерируемого измерительной катушкой при возбуждении структуры магнитным полем с фиксированной частотой $f_{s}=3.08 \mathrm{kHz}$. В отсутствие накачки в спектре присутствовала только составляющая с частотой $f_{s}$ малой амплитуды $u_{s}=u_{0}$, обусловленная прямой электромагнитной наводкой. При включении накачки с амплитудой $e=280 \mathrm{~V} / \mathrm{cm}$ в спектре появлялась составляющая с частотой $f_{p}$ амплитудой $u_{p}$. Амплитуда составляющей $u_{s}$ с частотой $f_{s}$ при этом значительно возрастала.

На рис. 3, $a$ приведена зависимость амплитуды составляющей $u_{s}$ от амплитуды поля накачки $e$. Видно, что с увеличением $e$ напряжение $u_{s}$ сначала медленно растет, а затем экспоненциально возрастает при приближении к пороговому полю $e_{t h} \approx 300 \mathrm{~V} / \mathrm{cm}$. Максимальный коэффициент усиления напряжения составлял $K=10 \lg \left(u_{s} / u_{0}\right) \approx 22$. При полях накачки выше порога $e>e_{t h}$ наблюдалась устойчивая генерация напряжения с частотой $f_{s}=3.08 \mathrm{kHz}$ и амплитудой $u_{g} \approx 100 \mu \mathrm{V}$.

На рис. $3, b$ приведена зависимость напряжения $u_{s}$ от магнитного поля $H$ в отсутствие электрической накачки $(e=0)$ и при поле накачки $e \approx 270 \mathrm{~V} / \mathrm{cm}$. В обоих случаях напряжение $u_{s}$ сначала растет с увеличением $H$, достигает максимума при определенном $H_{m}$, а затем падает до нуля при насыщении ФМ-слоя резонатора. Поле максимума $H_{m}$ возрастает от $\sim 1.3$ до $\sim 1.6 \mathrm{kA} / \mathrm{m}$ с увеличением накачки.

Для объяснения измерений воспользуемся результатами работы [7], где решена задача о параметрическом усилении колебаний пьезоэлектрической микробалки при накачке на удвоенной частоте. Параметрическое усиление описывалось уравнением для линейного гармонического осциллятора с переменной константой жесткости

$$
m \frac{\partial^{2} x}{\partial t^{2}}+\frac{m \omega_{0}}{Q} \frac{\partial x}{\partial t}+\left[k_{0}+k_{p}(t)\right] x=F(t) .
$$

Здесь $m$ - масса, $x(t)$ - амплитуда колебаний, $\omega_{0}=2 \pi f_{0}-$ собственная частота, $Q-$ добротность в отсутствие накачки, $k_{0}-$ константа жесткости, $k_{p}(t)-$ изменение константы жесткости, $F(t)-$ внешняя вынуждающая сила.

В нашем случае роль возбуждающей силы $F(t)$ выполняет магнитное поле $h(t)=h \cos \left(\omega_{0} t+\varphi\right)$, создаваемое возбуждающей катушкой, которое за счет магнитострикции $\lambda$ приводит к возбуждению магнитоакустических колебаний в резонаторе. Роль $x(t)$ играет механическая деформация $S(t)$. Эта деформация вследствие обратного эффекта (эластомагнитный эффект или эффект Виллари) приводит к изменению намагниченности резонатора $\delta M(t)=\delta M \cos \left(\omega_{0} t\right)$. Изменение намагниченности $\delta M$ регистрируется приемной катушкой. Изменение жесткости осциллятора $k_{p}(t)=\Delta k \cos \left(2 \omega_{0} t\right)$ вызывается электрическим полем накачки $e(t)=e \cos \left(2 \omega_{0} t\right)$ с удвоенной частотой, приложенным к слою PZT структуры.

Для подтверждения механизма параметрической накачки была измерена зависимость частоты $f_{2}$ колебаний структуры от постоянного поля $E$. Коэффициент перестройки частоты составил $\delta f / \delta E \approx 53 \mathrm{~Hz} \cdot(\mathrm{kV} / \mathrm{cm})^{-1}$, что соответствует изменению модуля Юнга структуры $Y$ в зависимости от поля $E$ с коэффициентом $\delta Y / \delta E \approx 0.27 \cdot 10^{10}\left(\mathrm{~N} / \mathrm{m}^{2}\right) \cdot(\mathrm{kV} / \mathrm{cm})^{-1}$. Таким образом, изменение жесткости $\Delta k$ пропорционально амплитуде электрического поля, приложенного к слою PZT, что согласуется с данными [8].

В результате решения уравнения (1) в [7] получена зависимость амплитуды колебаний от амплитуды модуляции константы жесткости при оптимальной разнице фаз между возбуждающей силой и накачкой $\varphi=\pi / 2$, которая в наших обозначениях имеет вид

$$
u_{s}(e)=u_{0} /\left(1-e / e_{t h}\right) .
$$

Здесь $u_{s}(e)$ и $u_{0}-$ амплитуды напряжения с измерительной катушки в режиме усиления и в отсутствие накачки, $e_{t h}-$ пороговая амплитуда поля накачки, при которой $u_{s}$ стремится к бесконечности. Сплошной кривой на рис. $3, a$ изображена зависимость $u_{s}(e)$, рассчитанная

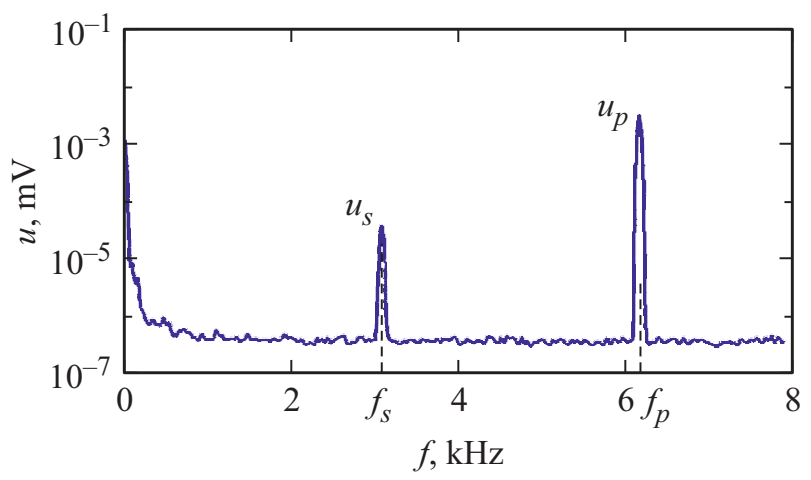

Рис. 2. Спектр частот напряжения с измерительной катушки при накачке $e=280 \mathrm{~V} / \mathrm{cm}$. 

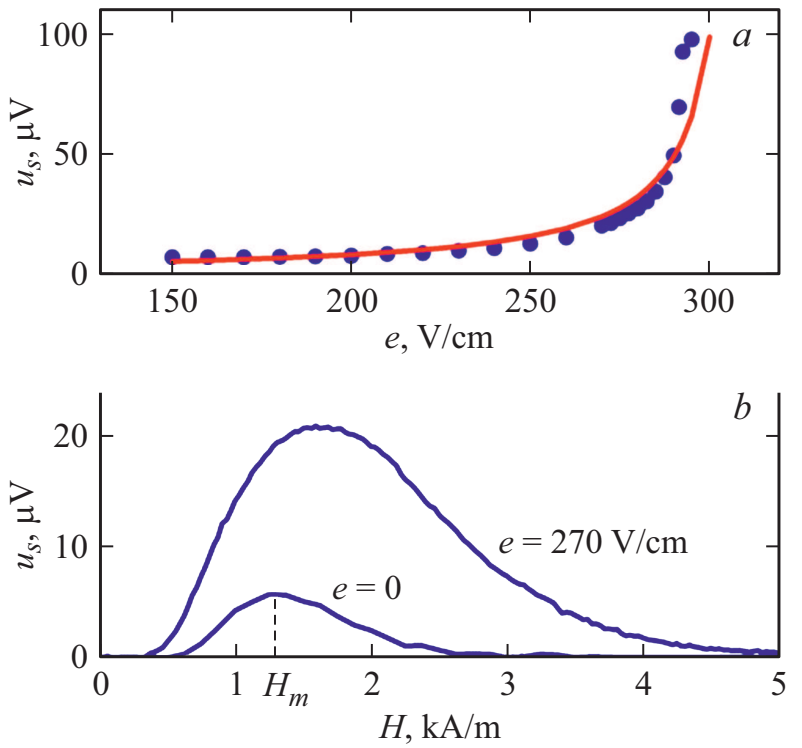

Рис. 3. Зависимости напряжения $u_{s}$ от поля накачки $e(a)$ и от поля $H$ при $e=0$ и в режиме усиления при $e=270 \mathrm{~V} / \mathrm{cm}$ (b).

по формуле (2) при значениях подгоночных параметров $u_{0}=3.3 \mu \mathrm{V}$ и $e_{t h}=310 \mathrm{~V} / \mathrm{cm}$. Видно, что теория хорошо описывает экспериментальные данные. При амплитудах поля накачки выше пороговой $e>e_{t h}$ резонатор переходит в режим параметрической генерации. Амплитуда генерируемого напряжения при этом ограничивается из-за нелинейных процессов в ФМ-слое, приводящих к сдвигу частоты резонанса [9]. В описанном резонаторе частота возбуждающего поля $f_{s}$ и частота поля накачки $f_{p}$ оказались расположены вблизи двух акустических резонансов структуры (рис. 1), что облегчило наблюдение вырожденного параметрического усиления при сравнительно небольших амплитудах поля накачки.

Зависимость амплитуды напряжения $u_{s}$ от постоянного поля $H$, приведенная на рис. $3, b$, обусловлена полевой зависимостью пьезомагнитного коэффициента ФМ-слоя $q(H)=\partial \lambda / \partial H$ (где $\lambda(H)$ - зависимость магнитострикционной деформации от поля), который определяет эффективность возбуждения магнитоакустических колебаний. Увеличение поля $H_{m}$, соответствующего максимуму пьезомагнитного коэффициента, с увеличением амплитуды колебаний согласуется с данными измерений при больших амплитудах возбуждающего поля [10].

Таким образом, в работе экспериментально обнаружено параметрическое усиление низкочастотных магнитоакустических колебаний в дисковом резонатоpe ферромагнетик-пьезоэлектрик. Усиление возникает вследствие изменения жесткости пьезоэлектрического слоя под действием электрического поля накачки с удвоенной частотой. Коэффициент усиления можно изменять с помощью постоянного магнитного поля, приложенного к структуре.

\section{Финансирование работы}

Работа поддержана Министерством науки и высшего образования РФ, проект МК-2639.2018.2, и Российским фондом фундаментальных исследований, грант 19-17-00594/19.

\section{Конфликт интересов}

Авторы заявляют, что у них нет конфликта интересов.

\section{Список литературы}

[1] Louisell W.H. Coupled mode and parametric electronics. N.Y.: Wiley, 1960. $268 \mathrm{p}$.

[2] Suhl H. // Phys. Rev. 1957. V. 106. P. 384-385.

[3] Hansryd J., Andrekson P.A., Westlund M., Lie J., Hedekvist P.O. // IEEE J. Sel. Top. Quant. Electron. 2002. V. 8. P. $506-520$.

[4] Dana A., Ho F., Yamamoto Y. // Appl. Phys. Lett. 1998. V. 72. P. 1152-1154. doi.org/10.1063/1.120998

[5] Tomas O., Mathieu F., Mansfield W., Huang C., TrolierMcKinstry S., Nicu L. // Appl. Phys. Lett. 2013. V. 102. P. 163504. doi.org/10.1063/1.4802786

[6] Wang Y., Onuta T.-D., Long C.J., Cheng Y., Takeuchi I. // Appl. Phys. Lett. 2015. V. 107. P. 192902. http://dx.doi.org/10.1063/1.4935332

[7] Rugar D., Grutter P. // Phys. Rev. Lett. 1991. V. 67. P. 699 702.

[8] Fetisov Y.K., Fetisov L.Y., Srinivasan G. // Appl. Phys. Lett. 2009. V. 94. P. 132507. doi.org/10.1063/1.3114406

[9] Fetisov Y.K., Burdin D.A., Ekonomov N.A., Fetisov L.Y., Berzin A.A., Hayes P., Quandt E. // Appl. Phys. Lett. 2018. V. 113. P. 022903. https://doi.org/10.1063/1.5032182

[10] Fetisov L.Y., Burdin D.A., Ekonomov N.A., Chashin D.V., Zhang J., Srinivasan G., Fetisov Y.K. // J. Phys. D: Appl. Phys. 2018. V. 51. P. 154003. DOI: 10.1088/1361-6463/aab384 